\title{
Quantitative Steinitz's Theorems with Applications to Multifingered Grasping*
}

\author{
David Kirkpatrick, ${ }^{1}$ Bhubaneswar Mishra, ${ }^{2}$ and Chee-Keng Yap $^{2}$ \\ ${ }^{1}$ Department of Computer Science, University of British Columbia, \\ Vancouver, Canada \\ ${ }^{2}$ Courant Institute of Mathematical Sciences, New York University, \\ New York, NY 10012, USA
}

\begin{abstract}
We prove the following quantitative form of a classical theorem of Steintiz: Let $m$ be sufficiently large. If the convex hull of a subset $S$ of Euclidean $d$-space contains a unit ball centered on the origin, then there is a subset of $S$ with at most $m$ points whose convex hull contains a solid ball also centered on the origin and having residual radius
\end{abstract}

$$
1-3 d\left(\frac{2 d^{2}}{m}\right)^{2 /(d-1)}
$$

The case $m=2 d$ was first considered by Bárány et al. [1]. We also show an upper bound on the achievable radius: the residual radius must be less than

$$
1-\frac{1}{17}\left(\frac{2 d^{2}}{m}\right)^{2 /(d-1)}
$$

These results have applications in the problem of computing the so-called closure grasps by an $m$-fingered robot hand. The above quantitative form of Steinitz's theorem gives a notion of efficiency for closure grasps. The theorem also gives rise to some new problems in computational geometry. We present some efficient algorithms for these problems, especially in the two-dimensional case.

* This research was supported by NSF Grants DCR-84-01898, DCR-84-01633, ONR Grant N00014-89-J3042, and NSF Grant Subcontract CMU-406349-55586. Chee-Keng Yap was supported in part by the German Research Foundation (DFG) and by the ESPRIT II Basic Research Actions Program of the EC under Contract No. 3075 (project ALCOM). 


\section{Introduction}

Carathéodory's theorem [2] states that given a subset $S$ of Euclidean $d$-space $\mathbb{E}^{d}$, any point inside its convex hull is also inside the convex hull of some subset of $S$ with at most $d+1$ points. Steinitz's theorem [13]-[15] states that given a subset $S \subseteq \mathbb{E}^{d}$, any point in the interior of its convex hull is also in the interior of the convex hull of some subset of $S$ with at most $2 d$ points. Bárány et al. [1] showed that the following quantitative version of Steinitz's theorem holds.

Theorem 1.1 (Quantitative Steinitz's Theorem). For any positive $d$ there is a constant $r=r(d)>d^{-2 d}$ such that, given any set $S \subseteq \mathbb{E}^{d}$ of points in $d$-space whose convex hull contains the unit ball centered at the origin o, there is a subset $X \subseteq S$ with at most $2 d$ points whose convex hull contains a ball centered at o with radius $r(d)$.

In fact, Bárány et al. [1] note that $r(d)>c(2 e d)^{-\llcorner d / 2\lrcorner d^{-2}}$ for some constant $c$. In this paper we generalize this quantitative Steinitz's theorem, and study various algorithmic questions related to it.

We introduce the following terminology: For any set $S \subseteq \mathbb{E}^{d}$, let the residual ball of $S$ refer to the largest (closed) ball $\mathbb{R}(S)$ centered at the origin $o$ such that the interior of $\mathbb{B}(S)$ is either fully outside or fully contained inside the convex hull of $S$. The residual radius of $S$, denoted $r(S)$, is the signed radius of this residual ball, where the sign is zero if $\mathbb{B}(S)$ is a point, otherwise the sign is positive or negative depending respectively on whether $\mathbb{B}(S)$ lies inside or outside the convex hull. Let $r_{d}(m, S)$ (or $r(m, S)$ if $d$ is clear from the context) denote the largest residual radius of a subset $X$ of $S$ with at most $m$ points. Let $r_{d}(m)$ denote the minimum value of $r_{d}(m, S)$, as $S$ ranges over all subsets $S \subseteq \mathbb{E}^{d}$ with $r(S) \geq 1$. Hence, for $m \geq 2 d$, the result of Bárány et al. shows that $d^{-2 d} \leq r_{d}(m) \leq 1$. Here we derive tighter upper and lower bounds for $r_{d}(m)$. Note that the notation $r(d)$ in the quantitative Steinitz's theorem above is simply the case of $r_{d}(2 d)$.

Application in Robotics. Our interest in these theorems comes from the study of robot hand grasps. We are interested in hands with $m$ frictionless (point-)fingers. A grasp in this model consists of $m$ points on the boundary of the body that we want to grasp. To grasp the body, we must then specify forces to be applied at these $m$ (grasp) points. A desirable notion of grasping is that of a closure grasp (see, for example, [9]-[11]). Intuitively, a closure grasp has the ability to respond to any external force or torque by applying appropriate forces at the grasp points. The quantitative Steinitz theorem gives us a measure of the efficiency of such closure grasps. Roughly speaking, the efficiency of the grasp is given by the ratio of largest external force-torque that can be resisted by applying at most unit forces at each of the grasp points; so a ratio of one corresponds to the most efficient grasp. The quantity $r_{d}(m)$ in the quantitative Steinitz's theorems gives this efficiency directly.

Computational Problems. These theorems naturally lead to new problems in computational geometry. For instance, given a finite set $S$ of points, and a number 
$m$, we want to find an $m$-subset of $S$ that achieves the residual radius $r(m, S)$. We will present algorithms for such problems. Here our strongest results are in two dimensions.

The rest of this paper is organized as follows. In Section 2 we explain more precisely the connection between quantitative Steinitz's theorem and grasping. Sections 3 and 4 prove the generalized Steinitz's theorems. Sections 5 and 6 present algorithms for computational versions of the generalized Steinitz's theorems. We conclude in Section 7.

\section{Application to Multifingered Positive Grasps}

We refer to [12] for a general survey of the field and to [8]-[11] for the theory of robot grasping as used in this work. Consider an idealized dextrous hand, consisting of $m>2$ independently movable force-sensing fingers. These fingers can only contact objects at their tips, and can thus be represented as points in three-dimensional space. The goal is to grasp a (closed, bounded, and connected) rigid object $K$. A finger can only apply a force on the object $K$ at the point of contact with $K$. We assume that the points of contact are nonsingular (i.e., the surface $\Sigma$ of $K$ has a unique surface normal at each such point) and frictionless, and hence the force can only be applied along the surface-normal at the point of contact, directed inward into the object $K$. An interesting task for such a hand is that of grasp selection for a given object $K$; by a grasp we mean a set of $m$ points on the boundary of $K$. We also describe such grasps in our model as positive because the fingers can only push into the body, but not pull at the body-as might happen if we postulate "sticky" fingers.

The object $K$ to be grasped is assumed to have a piecewise smooth boundary $\Sigma$. Assume that the grasp points are to be chosen from a given subset $S$ of the nonsingular points of $\Sigma$. For example, $S$ may consist of all nonsingular points of $\Sigma$ (by definition, the surface normals at nonsingular points are uniquely defined). Or again, $S$ may be a set of finitely many preselected points. For any point $\mathbf{r}$ in $S$, let $\mathbf{n}(\mathbf{r})$ denote the unit surface normal (directed inward) at $\mathbf{r}$. Define the function $\Gamma$ mapping $S$ into the six-dimensional force/torque space as follows:

$$
\begin{aligned}
\Gamma: S & \rightarrow \mathbb{R}^{6} \\
\mathbf{r} & \mapsto[\mathbf{n}(\mathbf{r}), \mathbf{r} \times \mathbf{n}(\mathbf{r})],
\end{aligned}
$$

where $x$ denotes the vector cross-product of three-dimensional vectors. Essentially, $\Gamma$ maps $\mathbf{r}$ to the point $\Gamma(\mathbf{r})$ in the force/torque space that represents the effects of applying a unit force at $\mathbf{r}$ in the direction $\mathbf{n}(\mathbf{r})$.

If $X \subseteq S$ is a set of $m$-points, we call $X$ an $m$-finger closure grasp if the interior of the convex hull of $\Gamma(X)=\{\Gamma(\mathbf{r}): \mathbf{r} \in X\}$ contains the origin $o$. It is shown in [11] that, for some $m \leq 12$, an $m$-finger closure grasp of $K$ exists if and only if 
Moreover, if $\Sigma$ is not a surface of revolution, the above condition is always satisfied with $S$ equal to the set of nonsingular points of $\Sigma$. For polyhedral objects, Mishra et al. [11] also gave an algorithm to find a 12-finger closure grasp in linear time. However, in the absence of any measure of "goodness" for closures grasps, the synthesized grasp may not be very robust. The motivation for our work is to quantify the goodness of closure grasps and to synthesize provably good closure grasps.

One criterion for goodness is the "efficiency" of a grasp, which measures the amount of external force and torque that can be resisted by applying at most a unit of force at each grasp point. This is precisely the value $r_{6}(m, \Gamma(S))$. To see this, note that if we choose $m$ points in $\Gamma(S)$ with residual radius $r$, then any force/torque vector $\mathbf{v}$ whose Euclidean norm is at most $r$ can be written as a convex combination of the $m$ chosen points. So if $\mathbf{v}$ is any external force/torque that is applied to the body $K$, and $v$ lies in the residual ball of radius $r$, we can counter this external force/torque by applying suitable forces (of magnitude at most 1) at the grasp points such that these forces sum to $-\mathbf{v}$; hence, we maintain the body in equilibrium.

These concepts can be specialized to the case where $K$ is a planar body in which case the force/torque space is three-dimensional. The number of fingers (12) for closure grasps can be reduced to six in this case [11].

\section{Quantitative Steinitz's Theorem in Two Dimensions}

Let $S \subseteq \mathbb{E}^{2}$ be a subset of the Euclidean plane and let $P$ be its convex hull. Without loss of generality, we may assume that $P$ has at least four vertices. Also, it is assumed that a unit disk $\mathbb{B}_{1}$ centered about the origin $o$ is contained inside $P$. In general, let $\mathbb{B}_{\varepsilon}$ denote the closed disk of radius $\varepsilon>0$ centered at the origin. Our goal is to develop techniques, given $S$ and $m$, for choosing a set $X$ of at most $m$ points from $S$ so that the residual radius of $X$ is maximized.

Lemma 3.1. Given $S$ as above, for any integer $k \geq 3$, we can find a subset $X$ of at most $2 k-1$ points of $S$ such that the convex hull of $X$ contains $\mathbb{B}_{\delta}$ with $\delta=\cos (\pi / k)$.

Proof. Take $k$ equally spaced rays from $o$, making sure that one of them passes through a vertex of $P$ (the convex hull of $S$ ). Let these rays intersect the unit circle centered at $o$ at the points $v_{1}, \ldots, v_{k}$. For each ray, if it intersects a vertex of $P$, then we choose that vertex and if it intersects an edge, we choose the two vertices of that edge. Thus we choose at most $2 k-1$ points of $S$, forming the subset $X \subseteq S$. Clearly, the convex hull of $X$ contains the points $v_{1}, \ldots, v_{k}$ and, hence, it contains the $\mathbb{B}_{z}$ with $\delta=\cos (\pi / k)$.

We now show that this bound is asymptotically tight. It is important to note that this bound is achieved by choosing vertices of the convex hull of $S$. 
Theorem 3.2. For all $m>4$, we have

$$
\frac{3 \pi^{2}}{2(m+1)^{2}}<1-r_{2}(m)<\frac{2 \pi^{2}}{m^{2}}
$$

Proof. The upper bound on $1-r_{2}(m)$ comes from the previous lemma which shows that $r_{2}(m)>\cos (2 \pi / m)$, and from the fact that $\cos x>1-x^{2} / 2$.

For the lower bound, we let $S$ be the vertices of a regular $(m+1)$-gon that just contains the unit disc $\mathbb{B}_{1}$. Then the omission of any point of $S$ gives a residual disk of radius

$$
\frac{\cos (2 \pi /(m+1))}{\cos (\pi /(m+1))}
$$

Thus $r_{2}(m)$ is upper bounded by this radius,

$$
\begin{aligned}
r_{2}(m) & <\frac{1-2 \pi^{2} /(m+1)^{2}+2 \pi^{4} / 3(m+1)^{4}}{1-\pi^{2} / 2(m+1)^{2}} \\
& =1-\frac{3 \pi^{2}}{2(m+1)^{2}-\pi^{2}}+\frac{4 \pi^{4}}{3(m+1)^{2}\left(2(m+1)^{2}-\pi^{2}\right)} \\
& <1-\frac{3 \pi^{2}}{2(m+1)^{2}}
\end{aligned}
$$

The special case where $m=4$ is of particular interest. We now give some special arguments for this. Starting with $S$ as above, the preceding lemma shows how to choose at most five points of $S$ whose convex hull contains the disk $\mathbb{B}_{\cos (\pi / 3)}=\mathbb{B}_{1 / 2}$. It is not hard to argue that one of these five points has the property that its two neighboring points span an angle of at most $144^{\circ}$ about the origin $o$ and hence if we delete this point, the remaining four points have a residual radius of at least $\left(\cos 72^{\circ}\right) / 2>0.15$. We can do better with the following argument.

\section{Theorem 3.3.}

$$
0.30<\sin 18^{\circ} \leq r_{2}(4) \leq \frac{\cos 72^{\circ}}{\cos 36^{\circ}}<0.38
$$

Proof. This upper bound is achieved by the regular pentagon (which is the special case $m=4$ of the proof in the previous theorem showing that, for $S$, given by the vertices of a regular $(m+1)$-gon, $r(m, S)=\cos (2 \pi /(m+1)) / \cos (\pi /(m+1)))$.

As before, let $P$ denote the convex hull of $S$. For the lower bound, fix $\beta$ to be any angle between $0^{\circ}$ and $60^{\circ}$. For any vertex $v_{0}$ of $P$, we define its forbidden zone which consists of two disjoint cones, each spanning an angle of $2 \beta$ at the origin $o$, and such that the two bisectors of these cones together with the ray $o v_{0}$ are equally spaced at $120^{\circ}$ apart. See Fig. 1. 


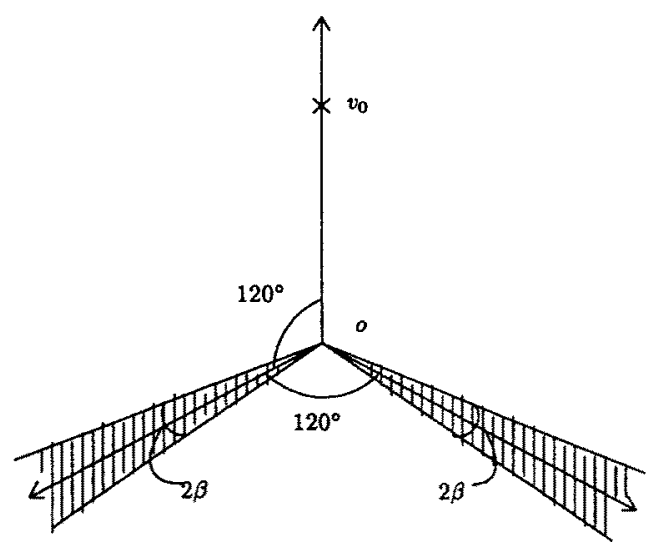

Fig. 1. Forbidden zone of $v_{0}$ is shaded.

A vertex $v_{0}$ of $P$ is bad if there is another vertex $v_{1}$ of $P$ that lies in its forbidden zone. First, if $v_{0}$ is bad, then we can choose three other vertices $v_{1}, v_{2}, v_{3}$ as follows. Let $v_{1}$ be a vertex of $P$ that lies in the forbidden zone of $v_{0}$. Let $R$ be the ray originating from $o$ and bisecting the larger of the two angles defined by the two rays from $o$ through $v_{0}$ and $v_{1}$, respectively. Let $v_{2}, v_{3}$ be the endpoints of the edge of $P$ that $R$ intersects. It is not hard to see that the quadrilateral $v_{0} v_{1} v_{2} v_{3}$ contains a circle of radius at least

$$
\sin \left(30^{\circ}-\frac{\beta}{2}\right)
$$

Now suppose that $P$ has no bad vertices. Assume that $v_{0}$ is vertically above $o$ and the two forbidden cones $C_{1}, C_{2}$ of $v_{0}$ are bounded by the rays $o \vec{R}_{1}, o \vec{R}_{2}$ and $o \vec{R}_{3}, o \vec{R}_{4}$, respectively, where the $R_{i}$ 's are points on the unit circle. Since $v_{0}$ is not bad, each ray in $C_{1}$ intersects a common edge of $P$, say, $v_{1} v_{2}$ and, similarly, each ray in $C_{2}$ intersects a common edge of $P$, say, $v_{3} v_{4}$. See Fig. 2 .

First suppose that the angle $\angle\left(v_{1} o v_{4}\right)$ (we always measure angles clockwise from $v_{1}$ to $v_{4}$ in this notation) is at most $\left(120^{\circ}+\beta\right)$. Then it is easy to see that the residual radius of $v_{1} v_{2} v_{3} v_{4}$ is at least $\sin \left(30^{\circ}-\beta / 2\right)$.

Hence assume that the angle $\angle\left(v_{1} o v_{4}\right)>\left(120^{\circ}+\beta\right)$. Without loss of generality, assume that $\angle\left(R_{1} o v_{1}\right) \leq \angle\left(v_{4} o R_{4}\right)$. Then we have

$$
\angle\left(R_{1} o v_{1}\right) \leq\left(60^{\circ}-\frac{3 \beta}{2}\right), \quad \angle\left(R_{3} o v_{1}\right) \leq\left(180^{\circ}-\frac{3 \beta}{2}\right) .
$$

Thus the distance from $o$ to the line through $v_{1}, R_{3}$ is at least $\sin (3 \beta / 4)$. It is easy to see that the distance from $o$ to the line through $v_{0}, v_{1}$ (resp. $v_{0}, R_{4}$ ) is at least $\sin \left(30^{\circ}+\beta / 2\right)$. The distance from $o$ to the line through $R_{3}, R_{4}$ is at least 1 . We 


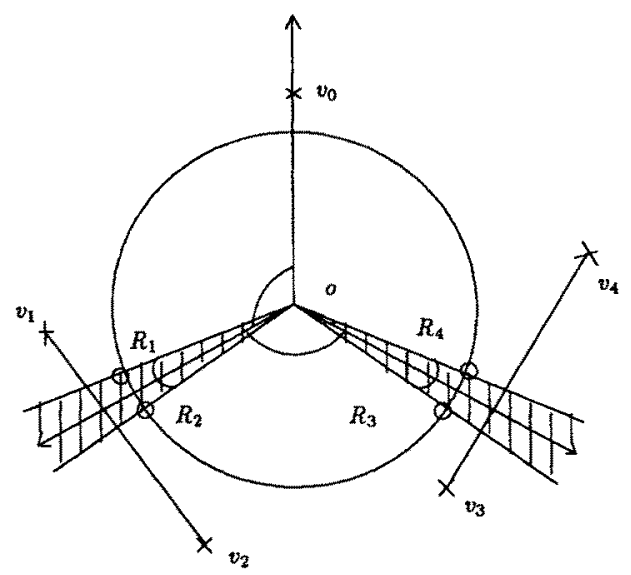

Fig. 2. Case of no bad vertices.

conclude that the residual radius of $v_{0} v_{1} v_{3} v_{4}$, which is at least the residual radius of $v_{0} v_{1} R_{3} R_{4}$, is at least

$$
\min \left\{\sin \left(30^{\circ}-\frac{\beta}{2}\right), \sin \frac{3 \beta}{4}\right\}
$$

where $0 \leq \beta \leq 60^{\circ}$. We choose $\beta=24^{\circ}$ (i.e., $30^{\circ}-\beta / 2=3 \beta / 4$ ) to maximize this expression. This proves our lower bound.

\section{Quantitative Steinitz's Theorems in Higher Dimensions}

We now consider the $d$-dimensional case for $d>2$. The techniques are slightly weaker than the two-dimensional case.

\subsection{Lower Bound}

We first give a lower bound for $r_{d}(m)$ for sufficiently large $m$ (in particular, for all $m \geq 13^{d} d(d+3) / 2$ ). Thus, $m$ is chosen to be large enough to guarantee that

$$
k=\left[\left(\frac{m}{2 d^{2}}\right)^{1 /(d-1)}\right]
$$

takes integral values greater than $\lceil 11 \sqrt{d}\rceil$.

Lemma 4.1. For any set $S \subseteq \mathbb{E}^{d}$ whose convex hull contains the unit ball $\mathbb{B}^{d}$ centered at the origin 0 , we can find a set $X \subseteq S$ of at most $m$ points with residual radius

$$
r(X) \geq 1-3 d\left(\frac{2 d^{2}}{m}\right)^{2 /(d-1)} \quad \text { for all } m \geq 13^{d} d^{(d+3) / 2}
$$


Proof. Let $k$ be defined as a function of $d$ and $m$, as before. It suffices to show that

$$
r(X) \geq 1-\frac{97}{48} \frac{d}{(k-1)^{2}}>1-\frac{3 d}{(k+1)^{2}}
$$

in the given range for $k$.

Henceforth, $P$ stands for the convex hull of $S$. Let $C$ be the $d$-dimensional cube whose faces are normal to the appropriate coordinate axes, of side-lingth 2 and containing the unit ball $\mathbb{B}^{d}$. On each face of $C$ we place a $k \times k \times \cdots \times k(d-1$ times) grid (so the grid points have coordinates that are integer multiples of $1 /(k-1)$ and two adjacent grid points are $2 /(k-1)$ apart $)$. Note that there are fewer than $2 d k^{d-1} \leq m / d$ "grid cubes" on the union of the $2 d$ faces of $C$. Through each grid point $p$, we pass a ray $R$ from the origin. Let $R$ intersect the unit sphere $S^{d-1}$ at $x(R)$. For each such ray $R$, we choose at most $d$ vertices of $P$ (the convex hull of $S$ ) as follows. If the ray passes through an $i$-face of $P$, we choose $i+1$ vertices of $P$ whose convex span intersects that ray and is contained in that $i$-face. Thus the set $X$ of chosen vertices has at most $m$ points. The convex hull of $X$ contains the set $X^{\prime}$ of all points of the form $x(R)$ where $R$ is a ray passing through the grid point.

Let $R$ be any ray originating from $o$ and suppose it intersects some face of $C$ at a point $a$ where $a$ lies inside a grid cube $S$. Consider the triangle $o a b$ where $b$ is any other point on the boundary of $S$ :

$$
\begin{aligned}
\sin \angle(a o b) & =|a b| \cdot \frac{\sin \angle(o a b)}{|o b|} \\
& \leq \frac{2 \sqrt{d}}{k-1} \cdot \frac{1}{1} \leq \frac{1}{5}
\end{aligned}
$$

Choose $\alpha$ to be

$$
\alpha=\arcsin \frac{2 \sqrt{d}}{k-1} .
$$

Let $q_{0}$ be any point at distance $\cos \alpha$ from the origin. We show that $q_{0}$ lies in the convex hull of $X^{\prime}$. Let $R_{0}$ be the ray from $o$ through $q_{0}$ and suppose $R_{0}$ intersects the grid cube $S_{0}$. Let $K_{0}$ be the cone bounded by the set of rays originating from $o$ that makes an angle of $\alpha$ with $R_{0}$. Hence each ray that passes through a vertex of $S_{0}$ is contained in $K_{0}$. There is a unique hyperplane $H_{0}$ containing $\partial\left(K_{0}\right) \cap S^{d-1}$. Note that $q_{0}=R_{0} \cap H_{0}$. Let

$$
T_{0}=\left\{x(R): R \text { passes through a vertex of } S_{0}\right\}
$$

and

$$
T_{1}=\left\{R \cap H_{0}: R \text { passes through a vertex of } S_{0}\right\}
$$


By definition, $T_{0} \subseteq X^{\prime}$. Note that each point in $T_{0}$ lies on the side of $H_{0}$ not containing the origin. This means that the convex hull of $X^{\prime}$ contains the set $T_{1}$, but the convex span of the set $T_{1}$ contains the point $q_{0}=R_{0} \cap H_{0}$. This proves $r(X) \geq r\left(X^{\prime}\right) \geq \cos \alpha$ :

$$
\begin{aligned}
\cos \alpha & =\left(1-\sin ^{2} \alpha\right)^{1 / 2} \\
& >1-\frac{\sin ^{2} \alpha}{2}-\frac{\sin ^{4} \alpha}{8} \sum_{i=0}^{\infty} \sin ^{2 i} \alpha \\
& =1-\frac{\sin ^{2} \alpha}{2}-\frac{\sin ^{2} \alpha}{8}\left[\frac{\sin ^{2} \alpha}{1-\sin ^{2} \alpha}\right] \\
& \left.\geq 1-\frac{97 \sin ^{2} \alpha}{192} \quad \text { (since } \sin ^{2} \alpha \leq \frac{1}{25}\right) \\
& \geq 1-\frac{97 d}{48(k-1)^{2}} .
\end{aligned}
$$

This proves the lower bound lemma.

\subsection{Upper Bound}

In this subsection we derive an upper bound for $r_{d}(m)$. For this purpose, we let $S$ be all the points on the unit sphere and then bound the largest radius of a ball contained in the convex hull of $m$ points on the unit sphere. The convex hull of any such $m$ points forms a polytope. The proof relies on the facts that

(1) any "long" edges of this polytope bound the radius of the contained ball and

(2) since the polytope has only $m$ vertices it must have some "long" edges.

The detailed calculations provide an appropriate numerical bound.

Lemma 4.2. Let $S \subseteq \mathbb{E}^{d}$ be the set of all points on the surface of the d-dimensional unit ball centered at the origin $o$. Thus, the convex hull of $S$ contains the unit ball $\mathbb{B}^{d}$ centered at the origin o. Then any set $X \subseteq S$ of at most $m$ points has a residual radius

$$
r(X) \leq 1-\frac{1}{17}\left(\frac{2 d^{2}}{m}\right)^{2 /(d-1)} \quad \text { for all } m \geq 3^{d} d^{2}
$$

Proof. The proof proceeds in two steps: We first show that, for all $m>0$ and for all $0<\Theta<\pi / 4$,

$$
r(X) \leq \max \left(\cos \frac{\Theta}{2}, 1-\frac{1-\tan ^{2} \Theta}{16}\left(\frac{2 d^{2}}{m}\right)^{2 /(d-1)}\right) .
$$


Then by an appropriate choice of the parameter $\Theta(\Theta=4 \pi / 53)$, we obtain the claimed bound.

(1) Let $X$ be a set of $m$ points in $\mathbb{E}^{d}$ all lying on the surface of a unit ball and $P=$ ConvexHull $(X)$. Let $P^{\prime}$ be the polyhedron obtained from $P$ by triangulating the nonsimplicial facets of $P$. Let $p q$ be an edge of the polyhedron $P^{\prime}$. Then

$$
r(X) \leq \cos \frac{\angle(p o q)}{2} .
$$

Thus, if

$$
\alpha=\max _{p q=\text { edge of } P^{\prime}} L(p o q)
$$

is the maximum of all such angles, then

$$
r(X) \leq \cos \frac{\alpha}{2} .
$$

If $\alpha \geq \Theta$, then

$$
r(X) \leq \cos \frac{\Theta}{2} .
$$

Henceforth, we assume that $\alpha<\Theta$. Let $t$ stand for $\tan \Theta$; thus $0<t<1$.

(2) Let $p \in X$ be any point, and define its truncated cone $K_{p}$ as follows:

$$
K_{p}=\{x: L(x o p) \leq \alpha \text { and } x \cdot p \leq 1\} .
$$

Now, if $q$ is an arbitrary point on the surface of the unit ball, then the line segment $o q$ belongs to $K_{p}$, for each vertex $p$ of some (simplicial) facet of $P^{\prime}$. As each such simplex facet has $d$ vertices, the collection of truncated cones cover each point in the unit ball at least $d$ times. Thus, we see that

$$
m \cdot \operatorname{Volume}\left(K_{p}\right) \geq d \cdot \operatorname{Volume}(\text { unit ball). }
$$

Let $V_{d}(r)$ stand for the volume of a $d$-dimensional ball of radius $r$ :

$$
V_{d}(r)=V_{d}(1) r^{d}
$$

Thus, the volume of the $d$-dimensional unit ball is given by

$$
\begin{aligned}
V_{d}(1) & =2 \int_{0}^{\pi / 2} V_{d-1}(\sin \theta) \sin \theta d \theta \\
& =2 V_{d-1}(1) \int_{0}^{\pi / 2} \sin ^{d} \theta d \theta \\
& =K(d) V_{d-1}(1),
\end{aligned}
$$


where $K(d)$ is defined by the last equation. The volume of each $K_{p}$ is given by

$$
\begin{aligned}
\operatorname{Volume}\left(K_{p}\right) & =\int_{0}^{1} V_{d-1}(r \tan \alpha) d r \\
& =V_{d-1}(\tan \alpha) \int_{0}^{1} r^{d-1} d r \\
& =\frac{V_{d-1}(\tan \alpha)}{d} .
\end{aligned}
$$

Substituting the volumes into the preceding inequality, we get

$$
m \frac{\tan ^{d-1} \alpha V_{d-1}(1)}{d} \geq d K(d) V_{d-1}(1) .
$$

Hence,

$$
1>t=\tan \Theta>\tan \alpha \geq\left(\frac{d^{2} K(d)}{m}\right)^{1 /(d-1)}=c(d, m)
$$

where $c(d, m)$ is defined in the last equation. Using the inequality $c(d, m)^{2}<t^{2}$, we get

$$
\cos ^{2} \alpha \leq \frac{1}{1+c(d, m)^{2}} \leq 1-c(d, m)^{2}+c(d, m)^{4} \leq 1-\left(1-t^{2}\right) c(d, m)^{2} .
$$

Hence,

$$
\cos \alpha=2 \cos ^{2} \frac{\alpha}{2}-1 \leq\left(1-\left(1-t^{2}\right) c(d, m)^{2}\right)^{1 / 2} \leq 1-\frac{1-t^{2}}{2} c(d, m)^{2}
$$

and

$$
\cos ^{2} \frac{\alpha}{2} \leq 1-\frac{1-t^{2}}{4} c(d, m)^{2}
$$

Finally, we get

$$
\cos \frac{\alpha}{2} \leq\left(1-\frac{1-t^{2}}{4} c(d, m)^{2}\right)^{1 / 2} \leq 1-\frac{1-t^{2}}{8} c(d, m)^{2} .
$$

Hence,

$$
r(X) \leq 1-\frac{1-t^{2}}{8}\left(\frac{d^{2} K(d)}{m}\right)^{2 /(d-1)}
$$


(3) Note that (e.g., p. 369 of [6])

$$
\begin{aligned}
K(d) & =2 \int_{0}^{\pi / 2} \sin ^{d} \theta d \theta \\
& = \begin{cases}\frac{(2 k-1) ! !}{(2 k) ! !} \pi & \text { if } d=2 k=\text { even, } \\
\frac{(2 k) ! !}{(2 k+1) ! !} 2 & \text { if } d=2 k+1=\text { odd }\end{cases} \\
& \geq 2\left(\frac{1}{2}\right)^{(d-1) / 2} .
\end{aligned}
$$

Here $k$ !! stands for $k(k-2)(k-4) \cdots(l+4)(l+2) l$ (terminating in $l=1$ or 2 , depending on whether $k$ is odd or even). Thus

$$
r(X) \leq 1-\frac{1-\tan ^{2} \Theta}{16}\left(\frac{2 d^{2}}{m}\right)^{2 /(d-1)} .
$$

(4) The stated bound follows with the appropriate choice of the parameter $\Theta$, as shown below: Let $m \geq 3^{d} d^{2}$; then

$$
\left(\frac{2 d^{2}}{m}\right)^{2 /(d-1)}<\frac{1}{9}
$$

Choose the parameter $\Theta=4 \pi / 53$, and observe that

$$
\cos \frac{2 \pi}{53}<1-\frac{1}{17 \times 9} \leq 1-\frac{1}{17}\left(\frac{2 d^{2}}{m}\right)^{2 /(d-1)} .
$$

Since $1-\tan ^{2}(4 \pi / 53)>16 / 17$

$$
1-\frac{1-\tan ^{2}(4 \pi / 53)}{16}\left(\frac{2 d^{2}}{m}\right)^{2 /(d-1)} \leq 1-\frac{1}{17}\left(\frac{2 d^{2}}{m}\right)^{2 /(d-1)} .
$$

If we choose $\Theta=\pi / 5$ in the preceding proof, we can show that, for all $m>0$,

$$
r(X) \leq 1-\frac{15}{512}\left(\frac{d}{m}\right)^{2 /(d-1)}
$$

If $m \leq d$, then

$$
0=r(X)<1-\frac{15}{512}\left(\frac{d}{m}\right)^{2 /(d-1)}
$$


On the other hand, if $m>d$, we get the result since $\cos \pi / 10<1-15 / 512$ and $1-\tan ^{2}(\pi / 5)>15 / 32$.

Summarizing Lemmas 4.1 and 4.2 ,

Theorem 4.3. For all $m \geq 13^{d} d^{(d+3) / 2}$,

$$
\frac{1}{17}\left(\frac{2 d^{2}}{m}\right)^{2 /(d-1)} \leq 1-r_{d}(m) \leq 3 d\left(\frac{2 d^{2}}{m}\right)^{2 /(d-1)}
$$

\section{Computational Problems in the Plane}

\subsection{Finding $m$ Vertices of a Convex Hull}

The quantitative Steinitz's theorem poses several interesting and new problems in computational geometry. We begin with the simplest version of such problems: given a convex $n$-gon $P$ whose interior contains the origin, find four vertices of $P$ whose residual radius is maximum. In this case we are able to give an elegant and simple linear-time method. Without loss of generality, we assume that $n \geq 5$ and the interior angles at each vertex of $P$ is less than $\pi$.

Theorem 5.1. There is a linear-time algorithm for finding a set $Q$ of four vertices of a convex $n$-gon, $P$, such that $Q$ has the maximum residual radius, $r(Q)=r_{2}(4, P)$.

We use the following general notations. Assume that the $u_{i}^{\prime} \mathrm{s}(i=1, \ldots, m ; n \geq 3)$ are points distinct from the origin. Let $\overrightarrow{o u}_{i}$ denote the ray from origin $o$ through $u_{i}$. The notation

$$
u_{1}>u_{2}>\cdots>u_{m}
$$

says that the rays $\overrightarrow{o u}_{i}$ 's are distinct and the ray $\overrightarrow{o u}_{i}$ is encountered before $\vec{u}_{i+1}$ when sweeping a ray originating from $o$ counterclockwise from $\overrightarrow{o u}$, to $\overrightarrow{o u}_{m}$. We extend this notation to the case where the $u_{i}$ 's are not necessarily distinct, but we still require that the rays $\overrightarrow{o u}_{1}$ and $\overrightarrow{u u}_{m}$ be distinct. For instance, we may write

$$
u_{1} \geq u_{2} \geq u_{3} \text { or } \quad u_{1}>u_{2} \geq u_{3}
$$

For any point $u$ on the boundary of the polygon $P$, let the successor succ $(u)$ of $u$ denote the vertex immediately following $u$ when we traverse the boundary of $P$ clockwise. If $u$ is a vertex of $P$, we insist that $\operatorname{succ}(u)$ is the next vertex of $P$.

Our algorithm is simple to describe-its correctness is slightly harder to see. Suppose that we have four vertices $u_{0}, u_{1}, u_{2}, u_{3}$ of $P$ such that there are at least three distinct vertices among them and

$$
u_{0} \geq u_{1} \geq u_{2} \geq u_{3}
$$


(The " $\geq$ " notation here makes sense since at most one of the inequalities is nonstrict.) Let $Q=u_{0} u_{1} u_{2} u_{3}$ denote the polygon formed by these vertices-so $Q$ is a triangle or a quadrilateral. Our goal is to choose one of these four points repeatedly, say $u_{i}$ for some $i=0, \ldots, 3$, to "advance," i.e., set $u_{i}$ to $\operatorname{succ}\left(u_{i}\right)$, in the hope of attaining a larger residual radius. The criteria for choosing the vertex to advance depends on the following two cases. Remark: Here, all arithmetic on subscripts are modulo 4.

$Q$ is a triangle. Suppose, for some $i=0, \ldots, 3, u_{i}$ and $u_{i+1}$ are coincident, that is $u_{i}=u_{i+1}$. Then we advance $u_{i}$ (the "forward vertex").

$Q$ is a quadrilateral. An edge $u_{i-1} u_{i}$ of the quadrilateral is limiting if the residual circle of $Q$ touches that edge. We then advance $u_{i}$ (the "backward vertex") where $u_{i-1} u_{i}$ is any such limiting edge.

We make some observations.

1. In case $Q$ is a triangle, advancing $u_{i}$ can in turn make $u_{i}$ and $u_{i-1}$ coincident, causing $u_{i-1}$ to be advanced in the next iteration. However, there cannot be more than three consecutive iterations in which $Q$ is a triangle. Note that a triangle $Q$ can have a nonpositive residual radius.

2. In case $Q$ is a quadrilateral and the residual radius $r(Q)$ is nonpositive, the limiting edge and hence $u_{i}$ is uniquely determined. After advancing $u_{i}$, provided $u_{i-1} u_{i}$ remains limiting, the radius $r(Q)$ will increase. This same vertex is repeatedly chosen, at least until the first time $t$ the edge $u_{i-1} u_{i}$ is no longer limiting. Observe that there are two possibilities at time $t:$ (a) $r(Q)$ becomes positive and (b) $r(Q)$ remains nonpositive. In the latter case, the edge $u_{i} u_{i+1}$ becomes limiting and we next start to advance $u_{i+1}$.

To complete the description of this algorithm we must initialize the four points and give the termination condition.

The Algorithm. Initially, we pick any four consecutive vertices of the polygon to serve as $Q=u_{0} u_{1} u_{2} u_{3}$. We record the initial position of $u_{0}$. Then we iterate the basic step of picking and advancing a $u_{i}$, updating if necessary the largest value of $r(Q)$ encountered so far. The algorithm halts when $u_{0}$ returns to its initial position, and outputs the largest value of $r(Q)$ recorded.

It is clear that the algorithm makes at most $4 n$ iterations when it halts.

For the next lemma we need some notations. Suppose $a_{0}, a_{1}, a_{2}, a_{3}$ are the vertices of $P$ that achieve the maximum residual radius $r^{*}=r_{2}(4, P)$. Without loss of generality, we may assume that all four vertices are distinct and

$$
a_{0}>a_{1}>a_{2}>a_{3}
$$

This partitions the vertices of the convex polygon into four nonempty sections, named $W_{0}, W_{1}, W_{2}, W_{3}$, where

$$
W_{0}=\left[a_{0}, a_{1}\right), \quad W_{1}=\left[a_{1}, a_{2}\right), \quad W_{2}=\left[a_{2}, a_{3}\right), \quad W_{3}=\left[a_{3}, a_{0}\right) .
$$




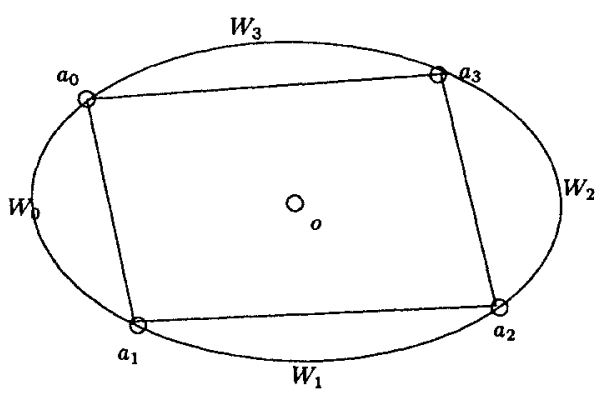

Fig. 3. $Q^{*}$ and four sections of the polygon $P$.

See Fig. 3. The notation $\left[a_{0}, a_{1}\right)$ refers to the consecutive subsequence of vertices from $a_{0}$ counterclockwise to (but not including) $a_{1}$. Also, let $Q^{*}=a_{0} a_{1} a_{2} a_{3}$.

The expression "at time $t, u_{j}$ is advanced from a vertex $a$ " means that "at time $t, u_{j}$ is at vertex $a$ and at time $t+1$ it is at $\operatorname{succ}(a) . "$

Lemma 5.2. Suppose, at time $t_{0}$, some $u_{j}(j=0, \ldots, 3)$ is advanced from $a_{i+1}$ of $Q^{*}$. (Hence at instant $t_{0}+1, u_{j}$ is in section $W_{i}$.) If $r^{*}$ is not yet attained by the algorithm by time $t_{0}$, then $u_{j-1}$ is not in $W_{i}$ at time $t_{0}$.

Proof. Without loss of generality, assume that $i=0=j$ in the statement of the lemma. That is, at time $t_{0}, u_{0}$ is advanced from vertex $a_{1}$. By way of contradiction, suppose $u_{3}$ is in $W_{0}$ at time $t_{0}$.

There are two cases.

Case 1. Suppose $Q$ is a triangle at time $t_{0}$. Let $t_{1}<t_{0}$ be the last instant when $Q$ was a quadrilateral. By a previous observation, $t_{0}-t_{1} \leq 3$. Note that at time $t_{1}$, for some $l=0, \ldots, 3, u_{l+1}$ is advanced so that $u_{l}$ and $u_{l+1}$ became coincident. Thus $u_{l}$ and $u_{l+1}$ are adjacent at time $t_{1}$. If $r(Q) \leq 0$ at time $t_{1}$, then the origin $o$ is on the side of the line $u_{l} u_{t+1}$ opposite to the other vertices of $P$, which is impossible. This shows that $r(Q)>0$. Since $Q$ is a quadrilateral, we only advance $u_{l} u_{l+1}$ because $u_{l} u_{l+1}$ is limiting, but $u_{l} u_{l+1}$ determines a radius greater than $r^{*}$, which leads to a contradiction.

Case 2. Suppose $Q$ is a quadrilateral at time $t_{0}$. Then $u_{3} u_{0}$ is limiting at time $t_{0}$. If $r(Q)$ were positive, we deduce that $r(Q)$ is at least $r^{*}$, which is a contradiction. If $r(Q)$ were nonpositive, then in order that $u_{3} u_{0}$ be limiting, the origin must lie to the left of the line directed from $u_{0}$ to $u_{3}$. This forces the origin to lie outside $Q *$, again leading to a contradiction.

We are now ready to show:

Lemma 5.3. The algorithm is correct.

Proof. Suppose the algorithm halts when $u_{0}$ returns to its original position $b_{0}$. Without loss of generality, assume that $b_{0}$ lies in the section $W_{3}=\left[a_{3}, a_{0}\right)$. Let $t_{0}$ 
be the instant when $u_{0}$ is advanced from $a_{0}$ (into $W_{3}$ ). If $r(Q)$ has already achieved the maximum value of $r^{*}$ before time $t_{0}$, then we are done. Otherwise we obtain a contradiction as follows. By the previous lemma, $u_{3}$ does not lie in $W_{3}$ at time $t_{0}$.

Claim. $u_{2}$ does not lie in $W_{2} \cup W_{3}$ at time $t_{0}$ : for if $u_{2}$ lies in $W_{3}$, then $u_{3}$ would be forced to be in $W_{3}$ as well; this is a contradiction. So it remains to show $u_{2}$ does not lie in $W_{2}$. If it does, then both $u_{2}$ and $u_{3}$ lie in $W_{2}$. Let $t_{1}<t_{0}$ be the last time that $u_{3}$ does not lie in $W_{2}$-such an instant is well-defined. So $u_{3}$ was advanced from $a_{3}$ at time $t_{1}$. Now an application of the previous lemma again shows that $r(Q)$ would have attained the maximum value $r^{*}$ before time $t_{1}$, which is a contradiction. This proves the claim.

We can repeat the argument of this claim to show that $u_{1}$ does not lie in $W_{1} \cup W_{2} \cup W_{3}$ at time $t_{0}$. Hence $u_{1}$ lies in $W_{0}$. Thus, both $u_{0}$ and $u_{1}$ lies in $W_{0}$. Again, let $t_{2}<t_{0}$ be the last time that $u_{1}$ does not lie in $W_{0}$. Then an application of the above lemma to $u_{1}$ at time $t_{2}$ yields the contradiction.

We easily extend the above method to finding the best $m \geq 4$ vertices of the polygon $P$. Now we need $O(\log m)$ per iteration (using a priority queue) to find the limiting edge of the current $m$-gon, and the number of iterations is at most $m n$. This yields the following theorem.

Theorem 5.4. For any $m \geq 4$ and $n \geq m$, there is an $O(n m \log m)$-time algorithm which on any input convex $n$-gon $P$ computes the value of $r_{2}(m, P)$.

\subsection{Finding $m$ Points in the General Case}

The above section considers algorithms to compute $r_{2}(m, S)$ for the special cases, where $S$ is the set of vertices of a convex polygon. In general, $S$ is an arbitrary set of points in the plane, and suppose $P$ is the subset of $S$ consisting of all the vertices of the convex hull of $S$. We note that $r_{2}(m, S)$ is in general larger than $r_{2}(m, P)$. As an example, let $P$ be the vertices of a regular pentagon and let $S$ contain, in addition to $P$, for each edge of the pentagon, a point in the interior of $P$ but lying very close to the mid-point of that edge. Then $r_{2}(4, S)>r_{2}(4, P)$.

On the other hand, $r_{2}(m, P)$ is a reasonably good lower bound to $r_{2}(m, S)$. This follows from our general constructions in Section 3 where the asymptotically tight lower bound for $r_{2}(m, S)$ is obtained by choosing points on the convex hull of $S$. Nevertheless, we may want to find the exact value of $r_{2}(m, S)$. This subsection gives such an algorithm. Again, we begin with the case $m=4$.

Theorem 5.5. There is an $O\left(n^{2} \log n\right)$ algorithm to find a set $X$ of four points in a set $S$ of $n$ points such that the residual radius of $X$ is maximized.

Let $S$ be a set of points with positive residual radius. Let the points of $S$ be assumed to be arranged by their (counterclockwise) angular order as in the previous subsection. We want to find four points in $S$ with the largest residual 


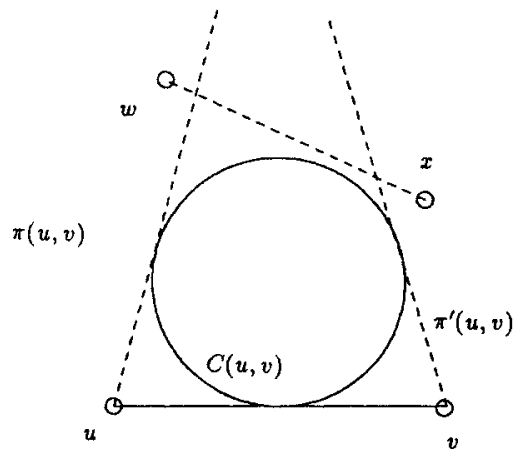

Fig. 4. A quadrilateral wuvx containing the circle $C(u, v)$.

radius. For any pair $u, v$ of distinct points, let $C(u, v)$ denote the circle centered at the origin which has the line $u v$ as tangent, and let $\operatorname{rad}(u, v)$ be the radius of $C(u, v)$. If, for some two points $w$ and $x$ of $S(w>u>v$ and $u>v>x), C(u, v)$ is contained in the quadrilateral wuvx, then the residual radius of $w u v x$ is equal to $\operatorname{rad}(u, v)$. (See Fig. 4.) (Note that it is possible that $w=x$.) In the remaining portion of this subsection we show how, for a given pair $u$ and $v$, such a choice of $w$ and $x$ (if they exist) can be made in logarithmic time, thus providing an $O\left(n^{2} \log n\right)$-time algorithm for the problem.

First we need some notations. Let $C$ be a circle and let $u$ and $v$ be points of $S$ outside $C$. For a given point $u$, let $\bar{u}$ denote the point diametrically opposite to $u$ (with respect to the origin $o$ ). We say $u$ and $v$ are mutually $C$-visible if the line segment connecting $u$ and $v$ does not intersect the interior of $C$. Also, we say $v$ is covered by $u$ (relative to $C$ ) if $v$ is mutually $C$-visible with $u$ and belongs to the smallest cone with its apex at $u$ and containing $C$. (See Fig. 5.) We say a point is relevant for $C$ if it is outside $C$ and not covered by any other points of $S$. We omit references to the circle $C$, if it is apparent from the context.

We have the following observations.

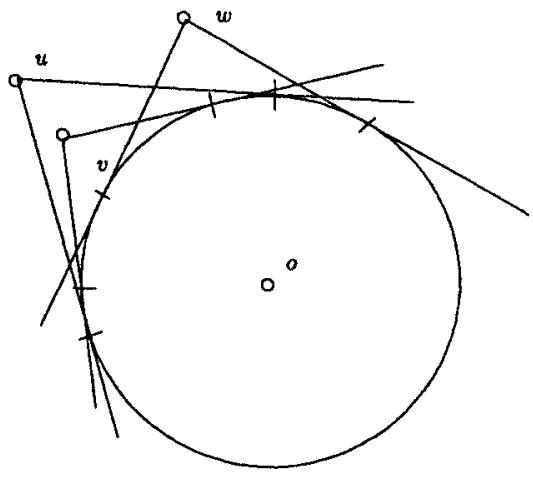

Fig. 5. $u$ covers $v$ but not $w$. $w$ covers neither $u$ nor $v$. 


\section{Lemma 5.6.}

1. Let wuvx be a convex quadrilateral containing $C(u, v)$. Consider two points $w^{\prime}$ and $x^{\prime}$ such that $w^{\prime}$ covers $w$ and $x^{\prime}$ covers $x$. Then one of the quadrilaterals, $w^{\prime} u v x^{\prime}$ or $x^{\prime} u v w^{\prime}$, also contains $C(u, v)$.

2. Let wuvx be a convex quadrilateral containing $C(u, v)$. Consider two relevant points $w^{\prime}$ and $x^{\prime}$ satisfying the following conditions: $\bar{u} \geq w^{\prime} \geq w \geq u ; v \geq$ $x \geq x^{\prime} \geq \bar{v} ; w^{\prime}$ and $u$ are mutually $C(u, v)$-visible and $x^{\prime}$ and $v$ are mutually $C(u, v)$-visible. Then either $w^{\prime} u v x^{\prime}$ or $x^{\prime} u v w^{\prime}$ is a convex quadrilateral and contains $C(u, v)$.

3. Let $C, C^{\prime}$ be two concentric circles with $C^{\prime}$ being the larger of the two. If $u$ is not relevant to $C$, then $u$ is also not relevant to $C^{\prime}$.

Let $u$ be a point outside $C$ and $W \subseteq S$ be the set of points $w_{1}, w_{2}, \ldots$ relevant to $C$ such that

$$
u \leq w_{1} \leq w_{2} \leq \cdots \leq \tilde{u}
$$

Let $w_{j}$ be a point in $W$ with the largest index such that,

$$
\text { for all } i=1,2, \ldots, j, \quad u \text { and } w_{i} \text { are mutually } C \text {-visible. }
$$

Then $w_{j}$ is the "rightmost" element in the set $W$, mutually visible with $u$. Suppose there is another element $w_{k} \in W(k>j)$ that is mutually visible with $u$. Then $k \geq j+2$ and $w_{j+1}$ is not mutually visible with $u$; in this case $w_{j+1}$ would be covered by $w_{k}$ thus contradicting the hypothesis that all points of $W$ are relevent. We say $w_{j}$ is the rightmost $C$-partner of $u$ (denoted $R P(u)$ ). Similarly, we may define the leftmost C-partner of $u(L P(u))$. We conclude that $R P(u)$ and $L P(v)$ can be computed (whenever they exist) in logarithmic time, if we have a balanced search structure that contains only the points relevant to $C(u, v)$ sorted by their angular order.

Now, using the preceding lemma, we observe that if, for some $w$ and $x$, the quadrilateral wuvx contains the circle $C(u, v)$, then so does one of the quadrilaterals $R P(u) u v L P(v)$ and $L P(v) u v R P(u)$. Thus it suffices to check that

(1) $C(u, v)$ is tangent to $u v$ at some point in the segment $u v$ and

(2) $R P(u)$ and $L P(v)$ are mutually $C(u, v)$-visible.

The basic idea is to put all vertices $u$, and also all unordered pairs $\{u, v\}$ of points in $S$, into a single priority queue. We use the Euclidean distance between $u$ and the origin $o$ as priority of $u$, and the value of $\operatorname{rad}(u, v)$ as priority of $\{u, v\}$. We may omit all $\{u, v\}$ 's with $\operatorname{rad}(u, v)=0$ and begin the processing of the queue by successively extracting items with the smallest priority. Note that we could assume that the first item extracted is a pair $\{u, v\}$.

For the first pair $\{u, v\}$ extracted from the queue, we need to initialize a data structure to store the points of $S$ relevant to $C(u, v)$ according to their angular order. We may break the circular ordering into a linear ordering at some arbitrary breakpoint. We store these points as a linear ordering in the leaves of a balanced binary tree $T$. 
In the general step, suppose we extract from the priority queue either a pair $\{u, v\}$ or vertex $u$. There are two cases.

Case 1. A pair $\{u, v\}$ is extracted and $C(u, v)$ touches $u v$ at some point $z$ in the segment $u v$. Then assuming $u \geq z \geq v$, we use the search tree $T$ to determine the rightmost $C(u, v)$-partner $w$ of $u$ and the leftmost $C(u, v)$-partner $x$ of $v$ and check if $w$ and $x$ are mutually $C(u, v)$-visible. If so, we have found a larger residual radius.

Case 2. (a) A pair $\{u, v\}$ is extracted and $C(u, v)$ touches $u v$ at some point outside the segment $u v$. Assume that of the two points $u, v$, the point $u$ is the closer to $o$. Then we note that for subsequent (larger radii) circles $C$, the vertex $v$ covers $u$ relative to $C$. Hence we can delete the element $u$ from the data structure $T$. (b) Similarly if $u$ is extracted, we can delete $u$ from $T$.

Clearly, each operation takes $O(\log n)$ time. Since there are $O\left(n^{2}\right)$ elements in the queue, the overall complexity is $O\left(n^{2} \log n\right)$. Note that at any instant when a pair $\{u, v\}$ is being considered, only relevant points of $S$ are left in $T$. More precisely, any point in the interior of $C(u, v)$ or covered by some other point would already have been deleted. (A point in the interior of $C(u, v)$ is at a smaller distance from $o$ than $\operatorname{rad}(u, v)$, and if $v^{\prime}$ covers $u^{\prime}$, then $\operatorname{rad}\left(u^{\prime}, v^{\prime}\right) \leq \operatorname{rad}(u, v) ; u^{\prime} v^{\prime}$ is touched by $C\left(u^{\prime}, v^{\prime}\right)$ outside the segment $u^{\prime} v^{\prime}$ and $u^{\prime}$ is closer to $o$ than $v^{\prime}$.) Thus it is clear that the algorithm is correct.

Again the method generalizes to finding any number $m$ of points that has the best residual radius. This yields the following theorem.

Theorem 5.7. For any $m \geq 4$ and $n \geq m$, there is an $O\left(n^{2} m \log n\right)$-time algorithm which, on any input set $S$ of $n$ points in the plane, computes the value of $r_{2}(m, S)$.

We note that a faster algorithm is possible if we are willing to settle for a good (to within a factor $\geq(1-\varepsilon), 0<\varepsilon<1$ ) approximation of $r_{2}(m, S)$, for all $m \geq 4$. Specifically, we can determine if $r_{2}(m, S)$ is $<$ or $\geq$ a fixed value $r$ in time $O(n m \log n)$. (In $O(n \log n)$ time we can determine the set of points that are relevant for a circle of radius $r$. For each relevant point $u$, in $O(m \log n)$ time, we can determine if there exist $\leq(m-1)$ other additional points such that the set containing $u$ together with these points has a residual radius of $r$ or larger.) To begin, we choose the best set of four vertices on the convex hull of $S$, and call its residual radius $r_{0}$. Using an $O(n \log n)$-time convex-hull algorithm, and the algorithm of the previous subsection, we guarantee that this step takes no more than $O(n \log n)$ time. Thus

$$
0.30<\frac{r_{0}}{r_{2}(m, S)} \leq 1
$$

Using $k=O(\log (1 / \varepsilon))$ comparisons, we can perform a binary search to improve the approximation to

$$
1-\varepsilon \leq \frac{r_{k}}{r_{2}(m, S)} \leq 1
$$


Thus, the resulting algorithm computes a good approximation (with a relative error of $\varepsilon)$ in time $O(n m \log n \log (1 / \varepsilon))$.

\section{Computational Problems in Higher Dimensions}

In this section we study the following algorithmic problem:

Given a set $S$ of $n$ points in $d$-dimensional Euclidean space, whose residual radius $r(S)$ is positive, find a subset $X \subseteq S$ of at most $m$ points such that the following inequality holds:

$$
\frac{r(X)}{r(S)} \geq \tilde{r}_{d}(m)=1-3 d\left(\frac{2 d^{2}}{m}\right)^{2 /(d-1)} .
$$

Here $m$ and $n$ are assumed to be sufficiently large, i.e., $n \geq m \geq 13^{d} d^{(d+3) / 2}$.

We do not discuss the more general "optimization" problem of finding a subset of $m$ points that maximizes the preceding ratio, for two reasons: firstly, for large $m$, the approximate solution provides a reasonably good answer; secondly, any hope for finding such a set in time polynomial in both $d$ and $n$ seems rather dim. While an investigation of this optimization problem is called for, we simply leave it as an open problem.

Returning to the stated problem, we see that this problem can be solved by essentially following the ideas outlined in Lemma 4.1: We first choose a set $X^{\prime}$ of at most $m / d$ points on the surface of the unit ball such that the residual radius of $X^{\prime}$ is no smaller than $\tilde{r}_{d}(m)$. We can then determine a set $X \subseteq S$ of at most $m$ points such that, for some $\lambda_{\min } \geq r(S)$, the convex hull of $X$ contains the set of points

$$
\lambda_{\min } X^{\prime}=\left\{\lambda_{\min } q: q \in X^{\prime}\right\}
$$

Thus

$$
r(X) \geq r\left(\lambda_{\min } X^{\prime}\right) \geq \lambda_{\min } \tilde{r}_{d}(m) \geq r(S) \tilde{r}_{d}(m) .
$$

The points of $X^{\prime}$ are chosen as follows; Let $C$ be the $d$-dimensional cube comprising the points $\left(x_{1}, \ldots, x_{d}\right)$ with $\left|x_{i}\right| \leq 1$ for $i=1, \ldots, d$. On each face of $C$, we place a $k \times k \times \cdots \times k((d-1)$ times) grid, with $k$ taking the value

$$
\left[\left(\frac{m}{2 d^{2}}\right)^{1 /(d-1)}\right] .
$$

Let

$$
X^{\prime}=\left\{o p \cap \mathbb{S}^{d-1}: p \text { is a grid point }\right\}
$$


Thus $\left|X^{\prime}\right| \leq 2 d k^{d-1} \leq m / d$. For each $q \in X^{\prime}$, we determine an appropriate set $S_{q} \subseteq S$ of at most $d$ points such that

$$
o q \cap \partial(\text { ConvexHull }(S)) \in \text { ConvexHull }\left(S_{q}\right)
$$

thus, for some $\lambda_{q}$,

$$
\lambda_{q} q \in \text { ConvexHull }\left(S_{q}\right)
$$

Let $X$ be

$$
X=\bigcup_{q \in X^{\prime}} S_{q}
$$

with $\lambda_{\min }$ taking the value $\min _{q \in X^{\prime}} \lambda_{q}$. Evidently, $\lambda_{\min } \geq r(S)$.

Note that $|X| \leq m$ and

$$
\lambda_{\min } X^{\prime} \subseteq \text { ConvexHull }(X)
$$

This demonstrates the correctness of the algorithm, since we know that the residual radius of $X^{\prime}$ is bounded from below by $\tilde{r}_{d}(m)$ (see the proof of Lemma 4.1).

In order to complete the algorithm, we show how to compute the set $S_{q}$ (for any point $q$ ) efficiently using the following linear-programming formulation. Let $S=\left\{p_{1}, p_{2}, \ldots, p_{n}\right\}$. Without loss of generality, we assume that the points of $S$ are in general position, i.e., at most $d$ points of $S$ may lie on any $(d-1)$-dimensional hyperplane. If not, the original points of $S$ may be perturbed using generic perturbation methods (see, for example, [16]); the following discussions still apply mutatis mutandis. Define the $d \times n$ matrix $\mathbf{A}$ whose $j$ th column consists of the coordinates of the point $p_{j}$. Corresponding to the point $q$, define a column $d$-vector b. The linear programming problem (LP) is given as follows:

- Given: A $d \times n$ matrix $\mathbf{A}$ and a column $d$-vector $\mathbf{b}$.

- Solve:

$$
\begin{aligned}
\operatorname{minimize}-\lambda & \\
\text { subject to } \quad \mathbf{A x} & =\lambda \mathbf{b}, \\
\mathbf{e}^{\mathrm{T}} \mathbf{x} & =1, \\
\mathbf{x} & \geq \mathbf{0}, \\
\lambda & \geq 0,
\end{aligned}
$$

where $\mathbf{x}=\left(x_{1}, \ldots, x_{n}\right)^{\mathrm{T}}, \mathbf{e}=(1, \ldots, 1)^{\mathrm{T}}$, and $\mathbf{0}=(0, \ldots, 0)^{\mathrm{T}}$ are column $n$ vectors. 
Let $x^{*}, \lambda^{*}$ be an optimal solution of (LP). Then $\lambda^{*}>0$ is the maximum value of $\lambda$ such that

$$
\lambda^{*} q=\sum_{i=1}^{n} x_{i}^{*} p_{i}
$$

with $\sum_{i=1}^{n} x_{i}^{*}=1$ and $x_{i}^{*} \geq 0$.

Now consider the following dual of the (LP), which will be referred to as (DLP):

$$
\begin{aligned}
& \text { maximize } y_{d+1} \\
& \text { subject to } a_{1,1} y_{1}+\cdots+a_{d, 1} y_{d}+y_{d+1} \leq 0 \text {, } \\
& a_{1,2} y_{1}+\cdots+a_{d, 2} y_{d}+y_{d+1} \leq 0 \text {, } \\
& a_{1, n} y_{1}+\cdots+a_{d, n} y_{d}+y_{d+1} \leq 0 \text {, } \\
& -b_{1} y_{1}-\cdots-b_{d} y_{d} \leq-1 \text {. }
\end{aligned}
$$

This problem can be solved in $O\left(3^{d^{2}} n\right)$ time by using Clarkson-Dyer's improvement on Megiddo's multidimensional search technique [4], [5], [7]. Let us now see how to recover the solution to the original problem.

Clearly both (LP) and (DLP) have optimal solutions. Let an optimal solution for (DLP) be

$$
\mathbf{y}^{*}=\left(y_{1}^{*}, \ldots, y_{d}^{*}, y_{d+1}^{*}\right)
$$

Let $I_{q} \subseteq\{1 \cdots n\}$ be the set of all the indices $j$ such that

$$
\mathbf{a}_{j} \cdot \mathbf{y}^{*}=a_{1, j} y_{1}^{*}+\cdots+a_{d, j} y_{d}^{*}+y_{d+1}^{*}=0
$$

where $\mathbf{a}_{j}=\left(a_{1, j}, \ldots, a_{d, j}, 1\right)^{\mathrm{T}}$. By the Complementary Slackness Theorem (see [3]), this implies that, for all $i=1, \ldots, n$, if $x_{i}^{*}>0$, then $i \in I_{q}$. By virtue of our nondegeneracy hypothesis about the points of $S$, we see that $\left|I_{q}\right| \leq d$. We now claim that $S_{q}=\left\{p_{j}: j \in I_{q}\right\}$ can serve as a desired solution. Clearly, $S_{q} \subseteq S$ has at most $d$ points and

$$
\left.o q \cap \partial(\text { ConvexHull }(S)) \in \text { ConvexHull }\left(S_{q}\right)\right)
$$

Note that even if the original set had been perturbed (by a sufficiently small amount), the set $S_{q}$ chosen from the unperturbed set $S$ still provides the desired solution.

To summarize: 
Theorem 6.1. For $n \geq m \geq 13^{d} d^{(d+3) / 2}$, we can find a set $X$ of at most $m$ points from an input set $S$ of $n$ points such that

$$
\frac{r(X)}{r(S)} \geq \tilde{r}_{d}(m)=1-3 d\left(\frac{2 d^{2}}{m}\right)^{2 /(d-1)},
$$

in time $O\left(3^{d^{2}} m n\right)$.

\section{Final Remarks}

It is natural to seek improved forms of Steinitz's theorem for certain subsets $S \subseteq \mathbb{E}^{d}$. In other words, if $k$ is any number (between $d+1$ and $2 d$ ), we want to characterize those subsets $S \subseteq \mathbb{E}^{d}$ whose residual radius is positive and are such that $S$ contains a subset $X$ of at most $k$ points, where $X$ has a positive residual radius. For instance, in the plane:

Lemma 7.1. Let $S \subseteq \mathbb{E}^{2}$ be any set with positive residual radius. Then there is a subset of three points in $S$ with positive residual radius if and only if $S$ is not contained in two lines through the origin.

We omit the easy proof. It would be interesting to develop an appropriate quantitative form of this lemma. We see that an obvious quantitative version for this lemma fails. That is, there does not exist a constant $0<\alpha<1$ with the following property:

Suppose the residual radius of $S \subseteq \mathbb{E}^{2}$ is at least one and $S$ does not lie in two lines through the origin. Then there exists three points in $S$ whose residual radius is at least $\alpha$.

To see this, consider the set $S=\{A, B, C, D, E\}$ where $A=(0,1), B=(1,0)$, $C=(-1,0), D=(1,-L)$, and $E=(-1,-L)$ for $L=L(\alpha)>0$ sufficiently large. Then no subset of $S$ with three points has residual radius at least $\alpha$.

Yet another area of research that calls for further investigation arises from the observation that the torque and force dimensions are really noncomparable. We want a notion of grasp-efficiency that can take this into account. A related issue is that the current approach depends on the origin of the reference frame in which the torques are measured. Is there an origin-independent approach to efficiency and other metrics of a grasp?

\section{Acknowledgments}

We are pleased to acknowledge some helpful discussions with Professor S. Rao Kosaraju of Johns Hopkins University and Professor Richard Pollack of the Courant Institute. We also thank an anonymous referee who pointed out a calculation error in the Lemma 4.2. 


\section{References}

1. I. Bárány, M. Katchalski, and J. Pach. Quantitative Helly-type theorems. Proc. Amer. Math. Soc., 86:109-114, 1982.

2. C. Carathéodory. Uber den Variabilitätsbereich der Koeffizienten von Potenzreihen, die gegebene Werte nicht annehmen. Math. Ann., 64:95-115, 1907.

3. V. Chvátal. Linear Programming. Freeman, New York, 1983.

4. K. L. Clarkson. Linear programming in $O\left(n 3^{d^{2}}\right)$ time. Inform. Process. Lett., 22:22-24, 1988.

5. M. E. Dyer. On a multidimensional search technique and its application to the Euclidean one center problem. Technical Report, Teeside Polytechnic, England, 1984.

6. I. S. Gradshteyn and I. M. Ryzhik. Table of Integrals, Series and Products. Corrected and enlarged edition. Academic Press, New York, 1980.

7. N. Megiddo. Linear programming in linear time when the dimension is fixed. $J$, Assoc. Comput. Mach., 31(1):114-127, 1984.

8. B. Mishra. Our exagmination of work in progress: tele and dextrous manipulation. Technical Report 194, Robotics Research Lab., Courant Institute of Mathematical Sciences, New York University, March 1989.

9. B. Mishra. Dexterous manipulation: a geometric approach. Proceedings of the Second International Workshop on Advances in Robot Kinematics, Linz, Austria, September 1990.

10. B. Mishra and N. Silver. Some discussion of static gripping and its stability. IEEE Trans. Systems Man Cybernet, 19:783-796, July/August 1989.

11. B. Mishra, J. T. Schwartz, and M. Sharir. On the existence and synthesis of multifinger positive grips. Algorithmica, 2:541-558, 1987.

12. J. Pertin-Troccaz. Grasping: a state of the art. In O. Khatib, J. J. Craig, and T. Lozano-Perez, editors, The Robotics Review 1. MIT Press, Cambridge, MA, 1989, pp. 71-98.

13. E. Steinitz. Bedingt Konvergente Reihen und Konvexe Systeme i. J. Reine Angew. Math., $144: 128-175,1913$

14. E. Steinitz. Bedingt Konvergente Reihen und Konvex Systeme ii. J. Reine Angew. Math., 144:1-40, 1914.

15. E. Steinitz. Bedingt Konvergente Reihen und Konvexe Systeme iii. J. Reine Angew. Math., 146:1-52, 1916.

16. C. Yap. A geometric consistency theorem for a symbolic perturbation scheme. J. Comput. System Sci., 40(1):2-18, 1990.

Received February 10, 1990, and in revised form November 30, 1990. 